\title{
Prevalence and patterns of coronary artery anomalies in 28,800 adult patients undergoing angiography in a large tertiary care centre in India
}

\author{
Ankit Bansal, Prattay Guha Sarkar, Mohit D. Gupta, MP Girish, Shekhar Kunal, Vishal Batra, \\ Jamal Yusuf, Safal, Saibal Mukhopadhyay, Sanjay Tyagi \\ Department of Cardiology, Govind Ballabh Pant Institute of Post Graduate Medical Education and Research, Delhi, \\ India
}

\begin{abstract}
Coronary artery anomalies (CAAs) are a diverse group of disorders with varied clinical presentation and pathophysiological mechanisms. A majority of these anomalies are asymptomatic and often an incidental finding on coronary angiogram or autopsy. This retrospective study included 28,800 patients who underwent coronary angiography from 2016 to 2020 . The coronary
\end{abstract}

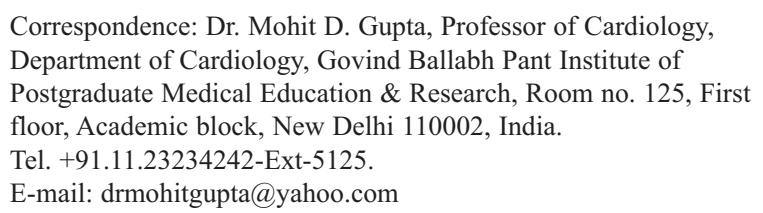

Contributions: AK, PGS, MDG, MPG, SK, collected the clinical data; SK, VB, JY, S, SM, ST, reviewed the literature; MDG, AK, worked on the concept and are responsible for the genuineness of the data. All the authors have drafted the manuscript and approved the final version of the manuscript and agreed to be accountable for all aspects of the work.

Conflict of interest: The authors declare that they have no competing interests, and all authors confirm accuracy.

Ethics approval and informed consent: This study was conducted in accordance with all relevant guidelines and procedures. Written informed consent obtained from all patients prior to the procedure.

Received for publication: 26 August 2021.

Accepted for publication: 16 December 2021.

Publisher's note: All claims expressed in this article are solely those of the authors and do not necessarily represent those of their affiliated organizations, or those of the publisher, the editors and the reviewers. Any product that may be evaluated in this article or claim that may be made by its manufacturer is not guaranteed or endorsed by the publisher.

${ }^{\circ}$ Copyright: the Author(s), 2021

Licensee PAGEPress, Italy

Monaldi Archives for Chest Disease 2022; 92:2066

doi: 10.4081/monaldi.2021.2066

This article is distributed under the terms of the Creative Commons Attribution-NonCommercial International License (CC BY-NC 4.0) which permits any noncommercial use, distribution, and reproduction in any medium, provided the original author(s) and source are credited. angiograms were reviewed by two independent reviewers and CAAs were documented. CAAs were classified into i) anomalies of coronary artery connection, ii) anomalies of intrinsic coronary arterial anatomy and iii) anomalies of myocardial/coronary artery interaction as proposed by the European Society of Cardiology. Of the 28,800 coronary angiograms, CAAs were present in $4.12 \%$ with anomalies in the left coronary artery (LCA) being most common. Anomalies of coronary artery connection were most common $(48.48 \%)$ followed by anomalies of myocardial/coronary artery interaction $(34.49 \%)$ and anomalies of intrinsic coronary artery anatomy (17.03\%). Among anomalies of coronary artery connection, absent left main trunk or split LCA with separate origins of left anterior descending coronary artery and left circumflex coronary artery from the left coronary sinus of Valsalva $(22.59 \%)$ was most common. An intramural course or "myocardial bridge" had an incidence of $1.16 \%$ while incidence of coronary artery fistulae (CAF) was $0.115 \%$.

\section{Introduction}

Coronary artery anomalies (CAAs) are a heterogeneous set of conditions with varied definition, morphogenesis, clinical presentation, diagnostic workup, prognosis, and treatment [1-5]. Though present at birth, most of these anomalies are often recognized as incidental findings during angiography. The prevalence of CAAs varies in different series ranging from 0.78 $5.64 \%$ in angiographic series $[2,3,6-9]$ to $0.99-5.79 \%$ on computed tomography series [10-12]. The pathophysiological mechanisms and clinical prognosis of various CAAs are different $[3,4]$. Though most of them are inconsequential, some of these can produce life-threatening symptoms like myocardial infarction, arrhythmias, syncope, and specifically sudden death in the young. Hence, identification of the various morphologies of CAA's is of utmost importance to predict the prognosis accurately. There is a scarcity of contemporary data reporting the patterns of CAAs in India. Hence, this study was designed to evaluate the incidence and contemporary patterns of CAAs in patients undergoing coronary angiography in the Indian population at a large tertiary care center.

\section{Methods}

This was a single centre retrospective study wherein the data regarding presentation patterns and coronary angiographic 
profile of 28,800 patients presenting a large tertiary care hospital in India from 2016 to 2020 was analysed. Indication for angiography in these patients included stable angina $(54.2 \%)$, acute coronary syndrome $(33.2 \%)$, valvular heart diseases $(11 \%)$ and others $(1.6 \%)$. The catheterization reports and angiograms were individually analyzed and reviewed by two independent reviewers and those with coronary anomalies were separated. These angiograms were further reviewed another set of two independent investigators before being classified. There was no interobserver variability among the independent investigators who had reviewed the coronary angiograms. In case of any difference of opinion, a consensus was reached after discussion. Coronary anomalies in patients undergoing catheterization for congenital heart disease were excluded. The anomalies were classified using criteria proposed by the European Society of Cardiology $[3,4]$, into anomalies of coronary artery connection, anomalies of intrinsic coronary arterial anatomy and anomalies of myocardial/coronary artery interaction (Figures 1 to 3 ). Data regarding the clinical details, symptomatology, coronary angiographic findings were entered in an Excel sheet. Baseline characteristics are expressed as mean ( \pm standard deviation) for normally distributed continuous variables and as frequency (\%) for categorical variables.

\section{Results}

Retrospective analysis of 28,800 consecutive angiograms revealed coronary anomalies in 1186 patients of whom $806(68 \%)$ were males. The mean age of the study group was $56 \pm 11$ years (range 21-87 years). Table 1 summarizes the incidence of coronary artery anomalies in the present series of 28,800 patients. The incidence of CAAs in our series was $4.12 \%$ with anomalies in the left coronary artery being most common. The anomalies of coronary artery connection were most common (48.48\%) followed by anomalies of myocardial/ coronary artery interaction (34.49\%) and anomalies of intrinsic coronary artery anatomy (17.03\%). Among the anomalies of coronary artery connection, absent left main trunk or split left coronary artery with separate origins of left anterior descending coronary artery (LAD) and left circumflex coronary artery (LCX) from the left coronary sinus of Valsalva (268 patients, $22.59 \%$ ) was the most common anomaly. Separate origins of LAD and LCX from the right coronary sinus of Valsalva were not observed in any patient. Anomalous origin of the LCX from the right sinus of Valsalva or RCA was the second most common anomalous connection, seen in $109(9.19 \%)$ patients. The right coronary artery arising from the left coronary sinus of Valsalva was

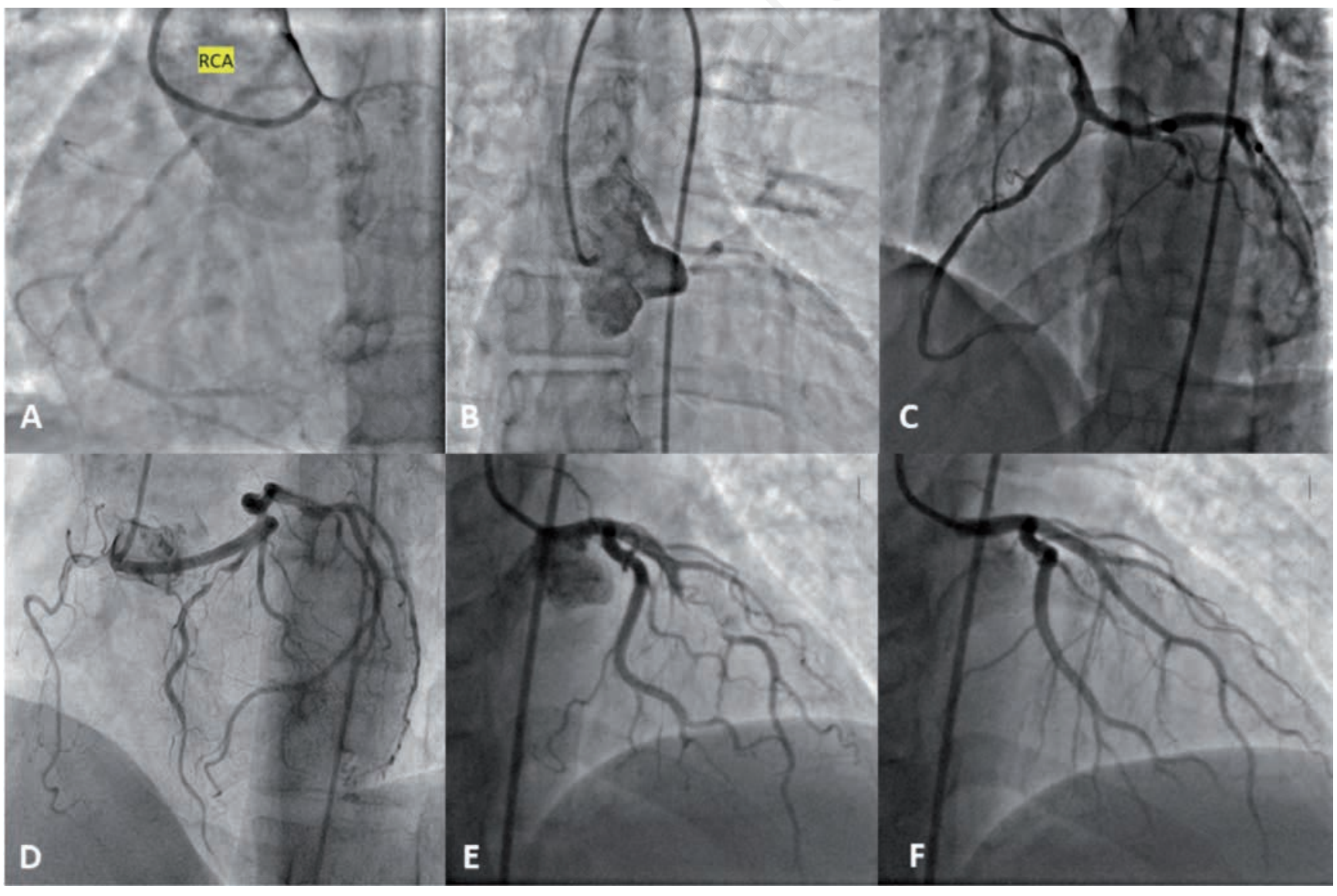

Figure 1. Coronary anomaly. A) Anomalous origin of RCA from the ascending aorta above the sino-tubular plane. B) Single coronary trunk arising from the ascending aorta above the sino-tubular plane. C) Selective engagement of the single coronary trunk arising from the ascending aorta with Amplatz Left-2 catheter. D) Single coronary trunk arising from the right coronary sinus with critical narrowing in LAD. E) Myocardial bridging in mid portion of LAD (systole). F) Myocardial bridging in mid portion of LAD (diastole). 
found in 54 patients $(4.55 \%)$. The left main trunk from the right sinus of Valsalva was found in 4 patients $(0.34 \%)$ while an isolated single coronary artery was seen in 24 patients $(2.02 \%)$. Anomalous left coronary artery from the pulmonary artery (ALCAPA) was present in $5(0.42 \%)$ patients.

Coronary artery ectasia was present in $25(2.1 \%)$ while coronary artery aneurysm were present in $31(2.61 \%)$ patients. In terms of anomalies of myocardial and coronary interaction, intramural course or myocardial bridge was the commonest with an incidence of $1.16 \%$ and it represented $28.16 \%$ of all CAAs. Of the 334 patients with myocardial bridge, 150 (44.9\%) were symptomatic with atypical chest pain $(72.1 \%)$ being the most common presenting symptom. Incidence of coronary artery fistulae (CAF) was $0.115 \%$ and they comprised $2.78 \%$ of all CAAs. The various CAAs in our series has been detailed in Table 2 . The most common presenting symptoms among patients with CAA were chest pain, dyspnea, palpitations, and syncope observed in $237(20 \%)$ cases. There was no difference in terms of clinical presentation among various CAAs however, patients with myocardial bridge had higher frequency of atypical chest pain while patients with CAF and ALCAPA presented with symptoms suggestive of heart failure. Among the patients with CAAs, atherosclerosis in anomalous vessels was observed in $386(32.6 \%)$ subjects while in those without CAAs, atherosclerosis was observed in $5136(18.6 \%)$ of the population. Selective cannulation of the aberrant arteries can be difficult and time-consuming. Since there are no specially defined catheters for every specific anomaly, we used a variety of catheters such as hockey-stick, multipurpose, right and left Amplatz, Judkins right and left guide catheters for coronary angiography in these cases.

Table 1. Overall incidence of coronary artery anomalies in the present study.

\begin{tabular}{lccc} 
& Number & Angiographic incidence (\%) Anomaly incidence (\%) & \\
Total coronary angiograms & 28,800 & 4.12 & 1.99 \\
Total coronary anomalies & 1186 & 575 & 0.70 \\
\hline Anomalies of coronary artery connection & 202 & 1.42 & 17.03 \\
Anomalies of intrinsic coronary artery anatomy & 409 & 34.49 \\
\hline Anomalies of myocardial/ coronary artery interaction & & \\
\hline
\end{tabular}

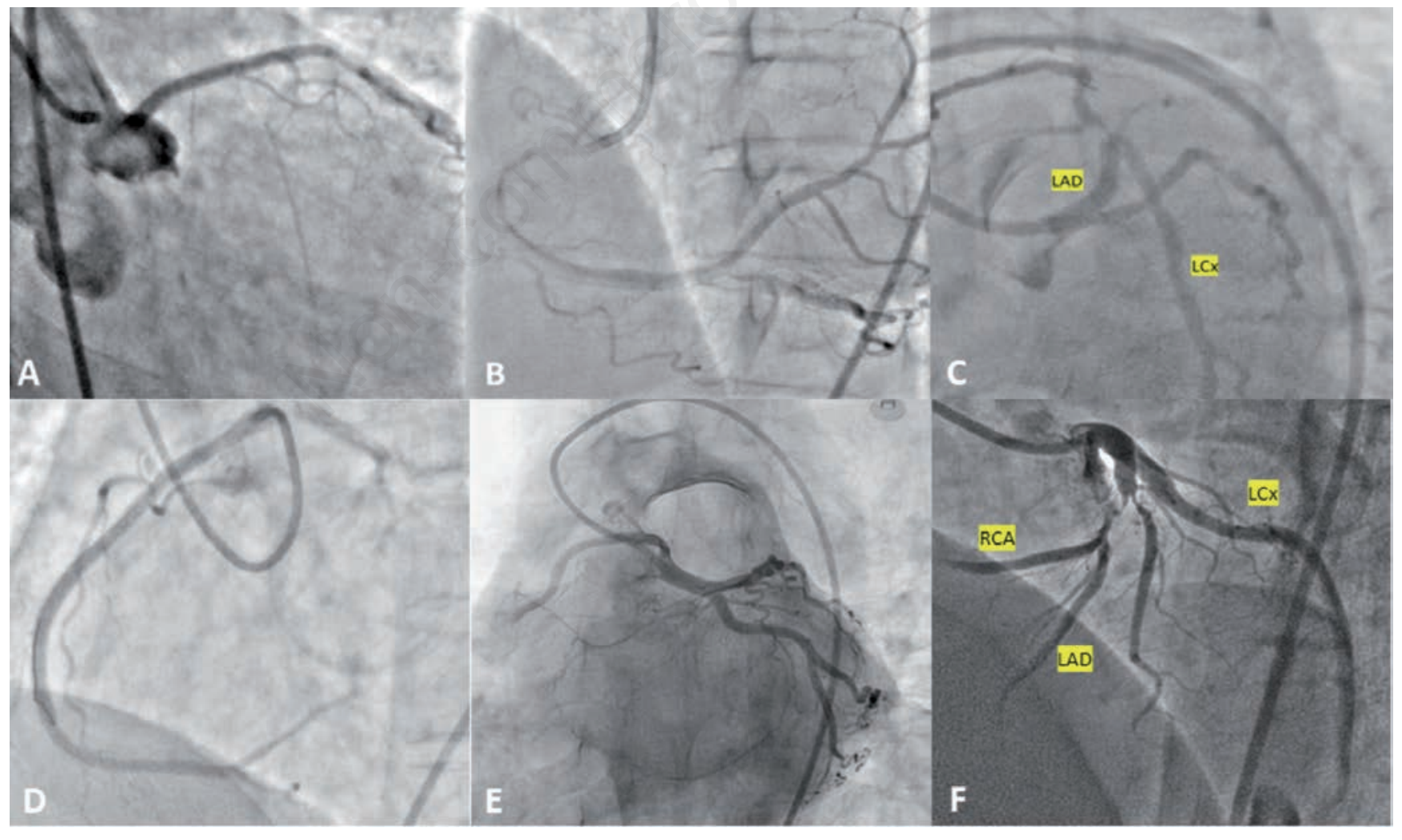

Figure 2. Coronary anomaly. A) Left circumflex artery ostial atresia. B) Posterolateral branch of RCA continuing as circumflex in the left AV groove. C) Left circumflex artery arising as first major branch of LAD. D) Anomalous origin of RCA from the left coronary sinus adjacent to the left coronary ostium. E) RCA arising as first major branch of left main coronary artery. F) RCA arising from the mid portion of LAD. 


\section{Discussion}

Most of the coronary artery anomalies are asymptomatic and are usually diagnosed incidentally at the time of coronary angiogram or autopsy. Normal coronary anatomy is defined as a feature which is present in $>1 \%$ of unselected sample and includes normal anatomical variants while anomalous coronary anatomy refers to morphological features present in $<1 \%$ of the population [3]. The prevalence of coronary anomalies shows a wide variation ranging from $0.78-5.64 \%$ in contemporary angiographic series $[2,3,6-9]$ to $0.99-5.79 \%$ in computed tomography based series [1012]. Such a wide variation in prevalence in various series can be explained by varied definitions of normal coronaries and CAAs. In the present series, we found a prevalence of $4.12 \%$ in patients undergoing diagnostic coronary angiography. In our series, the coronary angiograms were evaluated for anomalies as per the contemporary classification proposed by the ESC working group [4], which is a more comprehensive one.

Angina, dyspnea, palpitations, and syncope were the most common presenting features in the present study observed in 237 $(20 \%)$ cases. Earlier studies had reported these symptoms in $33 \%$ of patients [3]. In our study, the anomalies of coronary artery connection were most common (48.48\%) followed by anomalies of myocardial/coronary artery interaction $(34.49 \%)$ and anomalies of intrinsic coronary artery anatomy $(17.03 \%)$. Separate origin of LAD and LCX from left sinus or slit left coronary artery was the most common anomaly of coronary connection in our series (22.59\% of all coronary anomalies), but the incidence was slightly higher $(30.4 \%)$ in one of the largest series of 126,595 patients from Cleveland Clinic [13]. This anomaly is benign and the vessels are otherwise normal in distribution and course. The clinical importance lies in proper recognition at the time of diagnostic angiography and not to misinterpret as total occlusion or an anomalous origin of one branch. This anomaly can be well delineated with nonselective gentle contrast injection in the left anterior oblique (LAO) with caudal projection, where LAD will be arising more anteriorly and LCX will be arising posteriorly. LCX originating from the right sinus or RCA was the second most common anomaly of origin representing $9.19 \%$ of coronary anomalies. This

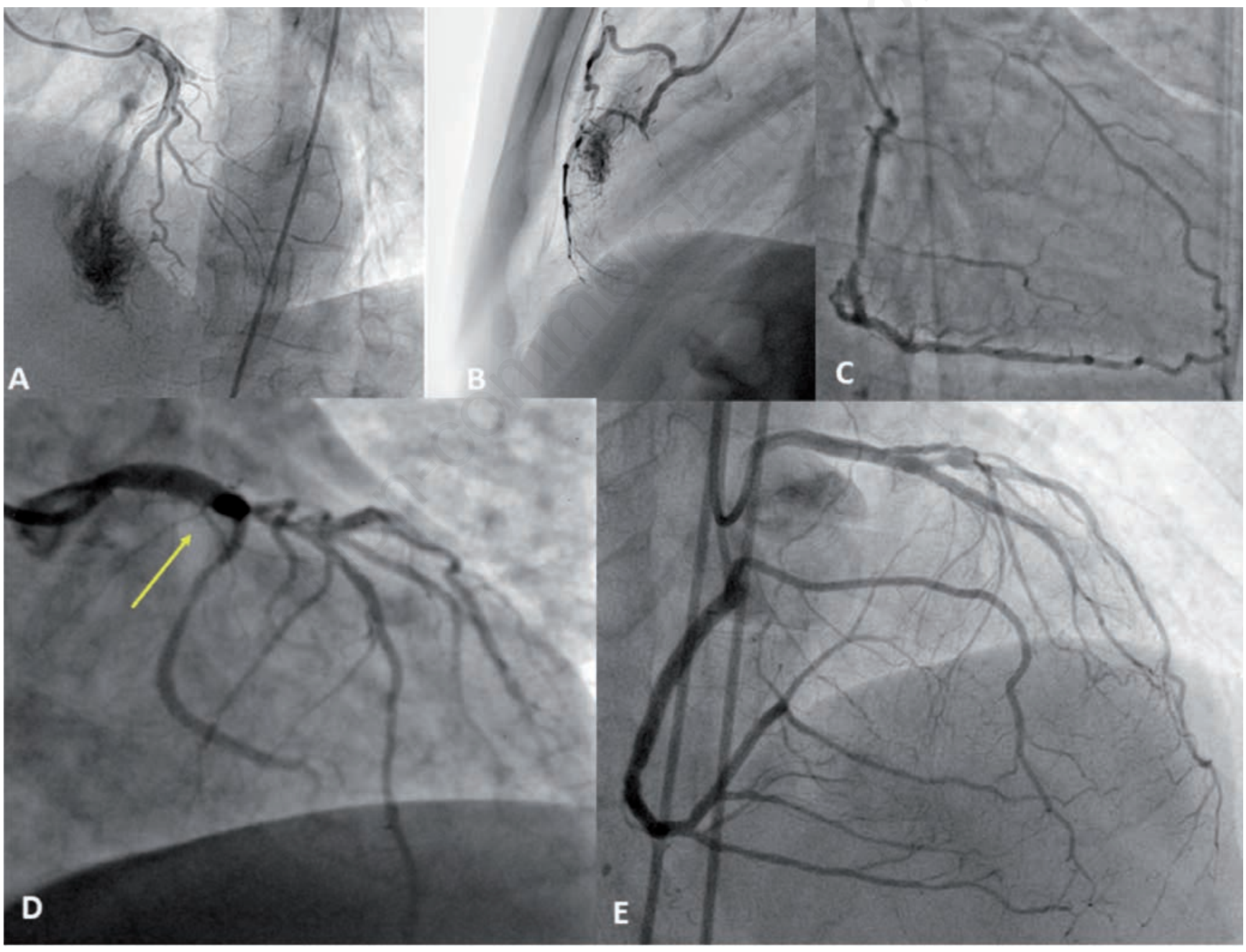

Figure 3. Coronary anomaly. A) Coronary cameral fistula arising from distal LAD and draining into right ventricle. B) Coronary cameral fistula arising from conus branch of RCA and draining into right ventricle. C) Right coronary injection showing the inter-coronary communication between RCA and LAD. D) Septal perforator arising as first branch of left main coronary artery. E) Simultaneous left and right coronary injection showing type IV dual LAD, with short LAD arising from left main coronary artery and long $\mathrm{LAD}$ arising from RCA. 
anomaly is thought to be clinically insignificant due to a dorsal course in relation to the left ventricle [4]. This anomaly can be frequently missed if the injection is from a catheter well intubated into RCA. LCX usually arises near the RCA ostium or separately from the right sinus as a posterior branch, hence catheter reposi- tioning is required accordingly. If intervention is required due to $\mathrm{CAD}$, the use of the Amplatz Right catheter provides selective engagement with support. RCA from the high posterior right sinus represented $8.6 \%$ of coronary anomalies and other than the challenge of selective engagement, this anomaly was relatively benign.

Table 2. Detailed distribution of coronary artery anomalies in 28,800 adult patients undergoing angiography in the present series.

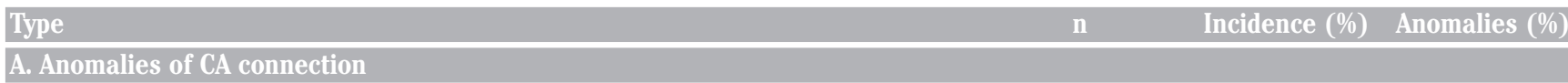

\section{a. to the pulmonary artery (PA)/pulmonary circulation}

1. LCA to posterior facing sinus (ALCAPA)

2. LCX to posterior facing sinus

3. LAD to posterior facing sinus

4. RCA to anterior right-facing sinus

5. Ectopic connection (outside facing sinuses) of any CA to PA left sinus, trunk, or branch 6. RV

b. to the aorta/systemic circulation

1. Absent left main trunk (split LCA)

2. Anomalous CA ostium location within or near proper aortic sinus of Valsalva

3. RCA to left sinus

4. LCA to right sinus

5. LCX to RCA/ or sinus

6. LAD to RCA/or sinus

7. RCA or LCA to posterior sinus

8. Origin of RCA from LMCA

9. Origin of LCX from mid LAD

10. Single CA

11. Anomalous CA ostium location outside sino-tubular aorta:

- LV

-Ascending aorta

50.017

$2 \quad 0.007$

0.42

0.007

0.17

- Aortic arch

- Others (innominate artery; right carotid artery; internal mammary artery; bronchial artery; subclavian artery; descending thoracic aorta)

B. Anomalies of intrinsic CA anatomy

1. CA ostial stenosis or atresia

2. CA ostial dimple

3. CA ectasia

4. CA aneurysm

5. Absent CA

6. CA hypoplasia

7. Anomalous CA ramification

- Anomalous origin of PD from LAD or septal penetrating branch

- Split RCA

- Split LAD

- Ectopic origin of first septal branch (RCA, right sinus, diagonal, LCX)

$-$

$-$

$-$

malous myocardial/CA interaction

1. Intramural course (myocardial bridge)

- Myocardial bridge LAD

- Myocardial bridge RCA

- Myocardial bridge LCX

2. Subendocardial course

3. Inter-coronary communications

4. Fistulae from RCA, LCA, or infundibular artery to: RV, RA, coronary sinus, superior vena cava,

PA, PV, LA, LV, multiple

- LAD-PA fistula

- LCX-PA fistula

- Conus branch-RV fistula

- RCA-RV fistula

- LCX-RV fistula

- RCA-RA fistula

2

$-$

25
31

$-$
0.930

0.187

0.014

0.378

0.354

0.003

0.010

0.083

0.010
22.59

4.55

0.34

9.19

8.60

0.08

0.25

2.02

0.25

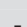

0.17

2.10

2.61

0.107
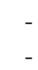

6.58

3.46

2.11

$\begin{array}{ll}41 & 0.142 \\ 25 & 0.087\end{array}$

0.142
0.087

$1.160 \quad 28.16$

$1.056 \quad 25.63$

$0.066 \quad 1.60$

$0.038 \quad 0.93$

$\begin{array}{lll}11 & 0.038 & 0.93\end{array}$

$\begin{array}{lll}42 & 0.140 & 3.54\end{array}$

0.115

$\begin{array}{ll}33 & 0.115 \\ 18 & 0.063\end{array}$

2.78

1.52

0.028
0.014

0.67

0.34

$0.003 \quad 0.08$

$0.003 \quad 0.08$

0.003

ALCAPA, Anomalous left coronary artery from the pulmonary artery; RCA, right coronary artery; LCX, left circumflex artery; LAD, left anterior descending artery; RV, right ventricle; RA, right atrium; PA, pulmonary artery; $\mathrm{LA}$, left atrium; LV, left ventricle; CA, coronary artery. 
The incidence of a single coronary artery was $0.083 \%$ and these represented $2 \%$ of all anomalies, quite similar to a large retrospective series [14]. The single CA may take the course of either an RCA or an LCA and divide shortly from its origin into two or three of the main coronary branches. Rarely, one of the major branches has an inter-arterial course with an increased risk of myocardial ischemia.

The anomalous origin of the LMCA from the right coronary sinus of Valsalva is a rare congenital coronary anomaly. This anomaly was identified in 4 cases $(0.014 \%)$, concurrent to those described in one of the largest series of 126,595 patients from Cleveland Clinic (0.017\%) [13]. The LMCA or LAD arising from the right sinus of Valsalva and RCA originating from the left sinus of Valsalva warrant clinical attention because these anomalies may be associated with sudden cardiac death in otherwise healthy individuals [15-18]. The anomalous origin of the LMCA from the right coronary sinus of Valsalva is further sub-classified based on the relationship of the LMCA to the great vessels: septal (beneath the right ventricular infundibulum), anterior to the pulmonary trunk, retro-aortic and inter-arterial (between the pulmonary trunk and the aorta) $[3,16,17]$. Reduction of the coronary blood flow resulting from compression over LMCA from the right coronary sinus of Valsalva by the pulmonary trunk or aorta during the inter-arterial course, due to pressure increase in these vessels upon exercise, further exacerbated by the acute takeoff or slit-like orifices of these arteries, produce ischemia resulting in angina, syncope, congestive heart failure, arrhythmias, or sudden death [3,16,17]. The exact anatomic location has been shown during catheterization utilizing the "dot and eye" method and/or placement of a catheter in the pulmonary artery [19]. Anomalous left coronary artery from the pulmonary artery (ALCAPA) is a rare but one of the more serious CAAs and is responsible for myocardial infarction and sudden cardiac death. Ischemia occurs due to coronary steal as retrograde flow occurs from the aorta through the abnormal LCA into the pulmonary trunk due to the low pressure in the pulmonary artery as compared to the aorta. The severity of presentation and clinical symptoms depend upon the extent of collateral formation. Outcomes are often poor in the "infant type" with less established collaterals while the "adult type" of the disease has a slight better prognosis owing to well developed collateral vessels [4]. In our series, ALCAPA was present in $0.42 \%$ of patients while in the largest series of CAAs, ALCAPA was present in $0.95 \%$ [13]. These patients often have an impaired cardiovascular mechanics with one of the studies reporting impaired left ventricular (LV) longitudinal deformation and LV torsion along with diastolic dysfunction following surgery in asymptomatic ALCAPA with normal global systolic function (LVEF $>50 \%$ ) [20].

Computed tomographic coronary angiography (CTCA) is currently regarded as the gold standard method for identification and visualization of CAAs. This is a safe and effective noninvasive imaging modality for defining coronary anomalies and provides detailed 3-dimensional anatomic information [21]. Additionally, CTCA offers better spatial resolution, lower acquisition time and good image contrast as compared to invasive coronary angiography (ICA). CTCA can delinate the course of anomalous coronary arteries and provide details regarding the relation with other cardiovascular structures. Conventional coronary angiography being an invasive modality caries a slight higher risk and provides two dimensional representation of the coronary vasculature. A higher prevalence of CAAs has been reported on CTCA as compared to ICA. In one of the series comparing CTCA with ICA, the overall prevalence of CAAs detected by CTCA was significantly higher than by ICA $(7.85 \%$ vs $2.02 \%$; $<0.01)$ [22].
Among the anomalies of myocardial and coronary interaction, the intramural course or myocardial bridge was the commonest with an incidence of $1.16 \%$ and represented $28.16 \%$ of all coronary anomalies in our series. In the historic autopsy data, the prevalence of myocardial bridge is much higher $15-85 \%$ as many myocardial bridges having slender myocardial fibers are often missed during conventional angiography [23]. Myocardial bridges were most commonly seen in LAD (25.63\%) and rarely can be seen in multiple coronaries or their branches [24]. When severe, myocardial bridges may produce angina and may predispose to atherosclerosis due to repeated trauma. The incidence of CAF in our study was $0.115 \%$ and they comprised $2.78 \%$ of all coronary anomalies. The incidence is around $0.12 \%$ in other angiographic series [13]. The most common type of fistula observed was the coronary to pulmonary artery type and usually arises from the LAD or the LCx artery. The size of the fistula, its tortuosity, and the site of drainage determine the hemodynamic significance. Diversion of blood from the coronary artery by the fistula may produce coronary steal and ischemia manifesting as angina, myocardial infarction, congestive heart failure, syncope, and sudden cardiac death [25].

The present study is one of the largest contemporary series utilizing the recent ESC classification for CAAs. Our study is among the foremost and the largest from the South-East Asian region to highlights the patterns and presentations of CAAs. Retrospective nature, single-center study, referral bias, and angiography in patients with signs and symptoms of a cardiovascular disease (symptomatic cases) leading to a bias are major limitations of the present series.

\section{Conclusions}

CAAs represents a heterogeneous group with diverse pathophysiology and clinical presentation. Though most of the anomalies are benign, it is important to identify anomalies associated with adverse outcomes like sudden cardiac death. A careful demonstration can assist in choosing appropriate hardware for percutaneous intervention and plan surgical care when needed.

\section{References}

1. Roberts WC. Major anomalies of coronary arterial origin seen in adulthood. Am Heart J 1986;111:941-63.

2. Angelini P, Villason S, Chan Jr. A, Diez JG. Normal and anomalous coronary arteries in humans. In: Angelini, P, editor. Coronary artery anomalies: A Comprehensive Approach. Philadelphia: Lippincott, Williams and Wilkins; 1999. pp 27150 .

3. Angelini P. Coronary artery anomalies: An entity in search for identity. Circulation 2007;115:1296-305.

4. Pérez-Pomares JM, de la Pompa JL, Franco D, et al. Congenital coronary artery anomalies: a bridge from embryology to anatomy and pathophysiology-a position statement of the development, anatomy, and pathology ESC Working Group. Cardiovasc Res 2016;109:204-16.

5. Frommelt P, Lopez L, Dimas VV, et al. Recommendations for Multimodality Assessment of Congenital Coronary Anomalies: A Guide from the American Society of Echocardiography: Developed in collaboration with the 
Society for Cardiovascular Angiography and Interventions, Japanese Society of Echocardiography, and Society for Cardiovascular Magnetic Resonance. J Am Soc Echocardiogr 2020;33:259-94.

6. Wilkins CE, Betancourt B, Mathur VS, et al. Coronary artery anomalies: a review of more than 10,000 patients from the Clayton Cardiovascular Laboratories. Tex Heart Inst J 1988; 15:166-73.

7. Kardos A, Babai L, Rudas L, et al. Epidemiology of congenital coronary artery anomalies: a coronary arteriography study on a central European population. Cathet Cardiovasc Diagn 1997;42:270-5.

8. Rigatelli G, Docali G, Rossi P, et al. Congenital coronary artery anomalies angiographic classification revisited. Int $\mathrm{J}$ Cardiovasc Imaging 2003;19:361-6.

9. Aydinlar A, Ciçek D, Sentürk T, et al. Primary congenital anomalies of the coronary arteries: a coronary arteriographic study in Western Turkey. Int Heart J 2005;46:97-103.

10. Duran C, Kantarci M, Durur Subasi I, et al. Remarkable anatomic anomalies of coronary arteries and their clinical importance: a multidetector computed tomography angiographic study. J Comput Assist Tomogr 2006;30:939-48.

11. Koşar P, Ergun E, Oztürk C, Koşar U. Anatomic variations and anomalies of the coronary arteries: 64-slice CT angiographic appearance. Diagn Interv Radiol 2009;15:275-83.

12. Cheng Z, Wang X, Duan Y, et al. Detection of coronary artery anomalies by dual-source CT coronary angiography. Clin Radiol 2010;65:815-22.

13. Yamanaka O, Hobbs RE. Coronary artery anomalies in 126,595 patients undergoing coronary arteriography. Cathet Cardiovasc Diagn 1990;21:28-40.

14. Desmet W, Vanhaecke J, Vrolix M, et al. Isolated single coronary artery: a review of 50,000 consecutive coronary angiographies. Eur Heart J 1992;13:1637-40.

15. Liberthson RR. Sudden death from cardiac causes in children and young adults. N Engl J Med 1996;334:1039-44.
16. Basso C, Maron BJ, Corrado D, Thiene G. Clinical profile of congenital coronary artery anomalies with origin from the wrong aortic sinus leading to sudden death in young competitive athletes. J Am Coll Cardiol 2000;35:1493-501.

17. Angelini P, Velasco JA, Ott D, Khoshnevis GR. Anomalous coronary artery arising from the opposite sinus: descriptive features and pathophysiologic mechanisms, as documented by intravascular ultrasonography. J Invasive Cardiol 2003; 15:507-14.

18. Angelini P, Walmsley RP, Libreros A, Ott DA. Symptomatic anomalous origination of left coronary artery from the opposite sinus of Valsalva: clinical presentations, diagnosis, and surgical repair. Tex Heart Inst J 2006;33:171-9.

19. Hejmadi A, Sahn DJ. What is the most effective method of detecting anomalous coronary origin in symptomatic patients? J Am Coll Cardiol 2003;42:155-7.

20. Di Salvo G, Siblini G, Issa Z, et al. Left ventricular mechanics in patients with abnormal origin of the left main coronary artery from the pulmonary trunk late after successful repair. Cardiology 2017;136:71-6.

21. Ghersin E, Litmanovich D, Ofer A, et al. Anomalous origin of right coronary artery: diagnosis and dynamic evaluation with multidetector computed tomography. J Comput Assist Tomogr 2004;28:293-4.

22. Ghadri JR, Kazakauskaite E, Braunschweig S, et al. Congenital coronary anomalies detected by coronary computed tomography compared to invasive coronary angiography. BMC Cardiovasc Disord 2014;14:81.

23. Angelini P, Trivellato M, Donis J, Leachman D. Myocardial bridges: a review. Prog Cardiovasc Dis 1983;26:75-88.

24. Gupta MD, Girish MP, Trehan V, Tyagi S. Myocardial bridging in all major epicardial vessels. JACC Cardiovasc Interv 2014;7:e129-31.

25. Levin D, Fellows KE, Abrams HL. Hemodynamically significant primary anomalies of the coronary arteries. Angiographic aspects. Circulation 1978;58:25-34. 